\title{
Negative Infinity
}

National Cancer Institute

\section{Source}

National Cancer Institute. Negative Infinity. NCI Thesaurus. Code C80215.

A number with a value that is less than any other negative number. 